\title{
Visual working memory as the substrate for mental rotation
}

\author{
Joo-Seok Hyun And STEven J. Luck \\ University of Iowa, Iowa City, Iowa
}

\begin{abstract}
In mental rotation, a mental representation of an object must be rotated while the actual object remains visible. Where is this representation stored while it is being rotated? To answer this question, observers were asked to perform a mental rotation task during the delay interval of a visual working memory task. When the working memory task required the storage of object features, substantial bidirectional interference was observed between the memory and rotation tasks, and the interference increased with the degree of rotation. However, rotation-dependent interference was not observed when a spatial working memory task was used instead of an object working memory task. Thus, the object working memory subsystem - not the spatial working memory subsystem - provides the buffer in which object representations are stored while they undergo mental rotation. More broadly, the nature of the information being stored - not the nature of the operations performed on this information - may determine which subsystem stores the information.
\end{abstract}

How is the representation of a visual object stored while mental rotation is performed? Although many studies have focused on the process of mental rotation, previous research has not considered how representations are stored while being rotated. In general, working memory is thought to be used for the temporary storage and manipulation of information, so it seems plausible that object representations are buffered in working memory during mental rotation. Some sort of buffer seems necessary to hold an intermediate representation of the object while it is being rotated because the original object remains visible and therefore controls the low-level sensory representations of the object.

Working memory for visual information can be stored in two dissociable subsystems: a spatial subsystem that stores spatial information and an object subsystem that stores nonspatial object features, such as color and form. This distinction is made on the basis of evidence from behavioral studies (reviewed by Logie, 1995), singleunit recordings (reviewed by Goldman-Rakic, 1996), and neuroimaging experiments (reviewed by Jonides et al., 2003). Although the process of mental rotation is spatial in nature and involves the dorsal stream (Carpenter, Just, Keller, \& Eddy, 1999; Gauthier et al., 2002; Jordan, Heinze, Lutz, Kanowski, \& Jancke, 2001), it is plausible that the storage of this object representation occurs in the object working memory subsystem. That is, because there is no evidence that the spatial working memory subsystem can represent detailed form information, it is more likely that representations of object form are held in the object working memory subsystem during mental rotation.
To test this hypothesis, we used a dual-task approach that has been used to explore the role of working memory in visual search tasks. Woodman, Vogel, and Luck (2001) asked subjects to perform a visual search task during the retention interval of a working memory task, and performance in this dual-task condition was compared with the performance observed when participants performed the memory and search tasks individually. The working memory task was a change detection task in which observers viewed an array of objects, retained the objects in working memory briefly, and were then presented with a test array that was either identical to the original array or different in the color or shape of one of the objects. Observers simply reported whether or not they detected a change. When the visual search task was performed during the retention interval of this working memory task, the efficiency of the search process was not impaired in comparison with when the visual search task was performed alone. That is, increases in the set size for the visual search task led to longer search reaction times (RTs), but the search rate (i.e., the slope of the RT $\times$ set size function) did not vary between the dual-task and single-task conditions of the experiment. Performance on the working memory task was slightly worse in the dual-task condition than when it was performed alone, but this effect was small, did not vary with the set size of the search task, and was caused by nonspecific masking of the memory stimuli. Thus, visual search and object working memory are largely independent. In contrast, Woodman and Luck (2004) found substantial and bidirectional interference when the search task was performed during the retention interval of a spa-

S. J. Luck, sjluck@ucdavis.edu 
tial working memory task. This finding is consistent with the hypothesis that spatial working memory is used to avoid returning to items that have already been searched (Castel, Pratt, \& Craik, 2003).

In the present study, we used this same approach, but replaced the visual search task with a mental rotation task. If the mental rotation target is stored in working memory while being rotated, then the rotation process should be slower and/or less accurate when it is performed during the retention interval of the working memory task than when it is performed alone. In addition, performance on the working memory task should become progressively less accurate as the degree of required rotation increases. We predicted that this pattern of interference would be obtained for an object working memory task (Experiment 1), but not for a spatial working memory task (Experiment 2).

\section{EXPERIMENT 1}

\section{Method}

Participants. Fifteen students between the ages of 18 and 30 received course credit for participating ( 3 male; 9 right-handed). All participants reported normal or corrected-to-normal visual acuity.

Stimuli and Procedure. The stimuli were viewed from $70 \mathrm{~cm}$ on a gray background. A light gray placeholder box $\left(6.1^{\circ} \times 6.1^{\circ}\right)$ was present at the center of the display, and the mental rotation letter was presented inside this box.

As Figure 1A illustrates, each trial began with a 500-msec presentation of a sample array consisting of four colored squares $\left(1.4^{\circ} \times\right.$ $1.4^{\circ}$ ), each centered $2^{\circ}$ diagonally from a corner of the placeholder box. Seven colors were used for the squares: black, white, red, green, blue, yellow, and violet. On each trial, the colors of the four squares were selected at random from this set with the constraint that no more than two squares could be the same color. After a $500-\mathrm{msec}$ delay, a black mental rotation letter $\left(2^{\circ} \times 2.5^{\circ}\right)$ was presented for $2,000 \mathrm{msec}$. The letter was selected at random from the set $\{\mathrm{F}, \mathrm{J}$, $\mathrm{G}, \mathrm{P}, \mathrm{R}, \mathrm{Q}, \mathrm{a}, \mathrm{h}, \mathrm{k}, \mathrm{t}\}$, and it was presented in a canonical or mirrorreversed form, rotated by $0^{\circ}, 72^{\circ}$, or $144^{\circ}$ from an upright position. Each combination of letter form and rotation angle occurred with equal probability. After a 500-msec delay, a test array was presented for $1,500 \mathrm{msec}$. This array was identical to the sample array, except that one of the colors was replaced by a different randomly selected color on $50 \%$ of trials. The next trial began after a $1,500-$ msec delay.

In the mental rotation task, participants made a speeded response with the index or middle finger of the dominant hand to indicate a canonical or mirror-reversed form, respectively. In the memory task, participants made an unspeeded response with the index or middle finger of the nondominant hand to indicate a change or no-change test array, respectively.

Each participant received three blocks of 48 trials in counterbalanced order. In the rotation-alone block, they performed the letter task but ignored the memory task. In the memory-alone block, they performed the memory task but ignored the letter task. In the dualtask block, they performed both tasks. Participants were required to perform a concurrent articulatory suppression task in which they continuously repeated "one, two, three" throughout each trial; this effectively discourages participants from using verbal memory (Baddeley, 1986; Besner, Davies, \& Daniels, 1981; Murray, 1968).

Responses with a latency of $<100 \mathrm{msec}$ or $>3,000 \mathrm{msec}$ were excluded from all analyses, and the RT analyses excluded trials with incorrect mental rotation responses. In addition, RTs in the rotation task were excluded if they fell more than two standard deviations from the mean for that condition. Memory accuracy was quantified as percent correct, collapsing across change and no-change trials (comparable results were obtained when the $d^{\prime}$ measure of sensitivity was used). Accuracy and RT for the rotation task were collapsed across canonical and mirror-reversed letter forms. Memory accuracy, rotation accuracy, and rotation RT were analyzed in separate two-way repeated measures ANOVAs with factors of condition (single-task vs. dual-task) and degree of rotation.

\section{Results and Discussion}

Figure 2A summarizes the results. The rate of mental rotation (i.e., the slope of the function relating rotation angle to mean RT) was $530 \%$ sec, and the main effect of rotation angle was significant $[F(2,28)=48.86, p<.001]$. The rotation rate was nearly identical in the rotation-alone and dual-task conditions, and the condition $\times$ rotation interaction did not approach significance $(F<1)$. Overall mental rotation RTs were approximately $85 \mathrm{msec}$ slower in the dual-task condition than in the rotation-alone condition, resulting in a significant main effect of condition $[F(1,14)=8.38, p<.05]$.

More substantial interference effects were observed in mental rotation accuracy. Accuracy declined significantly as the rotation angle increased $[F(2,28)=13.19$, $p<.001]$, and the magnitude of this decline was greater for the dual-task condition than for the rotation-only condition, yielding a significant condition $\times$ rotation angle interaction $[F(2,28)=8.50, p<.01]$.

Interference was also observed in the memory task. Specifically, memory accuracy was significantly lower in the dual-task condition than in the memory-alone condition $[F(1,14)=26.50, p<.001]$. Moreover, this effect became larger as the degree of rotation increased, yielding a significant condition $\times$ rotation angle interaction $[F(2,28)=4.29, p<.05]$.

These results provide clear evidence that mental rotation and object memory storage systematically interfere with

"one, two, three, one, two, three, one, two ..."

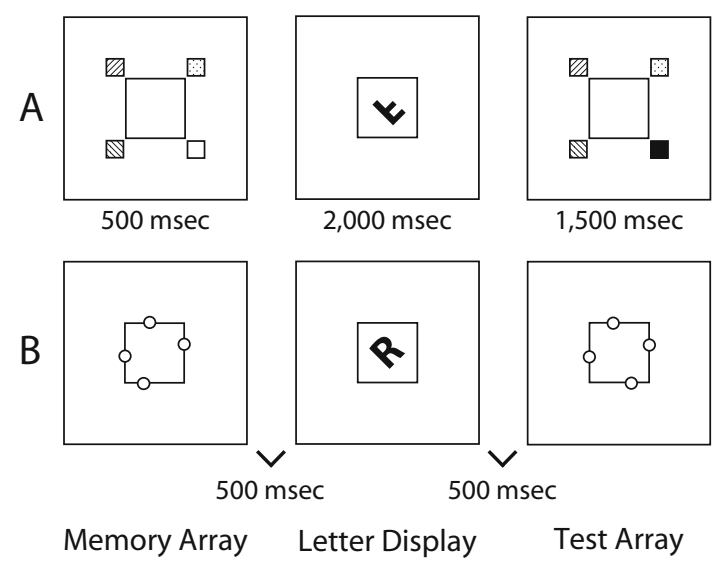

Figure 1. Example stimuli and procedures used in Experiments 1 and 2. (A) Stimuli and procedure used in Experiment 1. Squares filled with patterns represent the colored items. (B) Stimuli and procedure used in Experiment 2. Dots were colored in white, and the placeholder box at the center was outlined in a light gray. The stimuli were presented on a gray background. The text at the top represents the concurrent articulatory suppression task that was used in both experiments. 

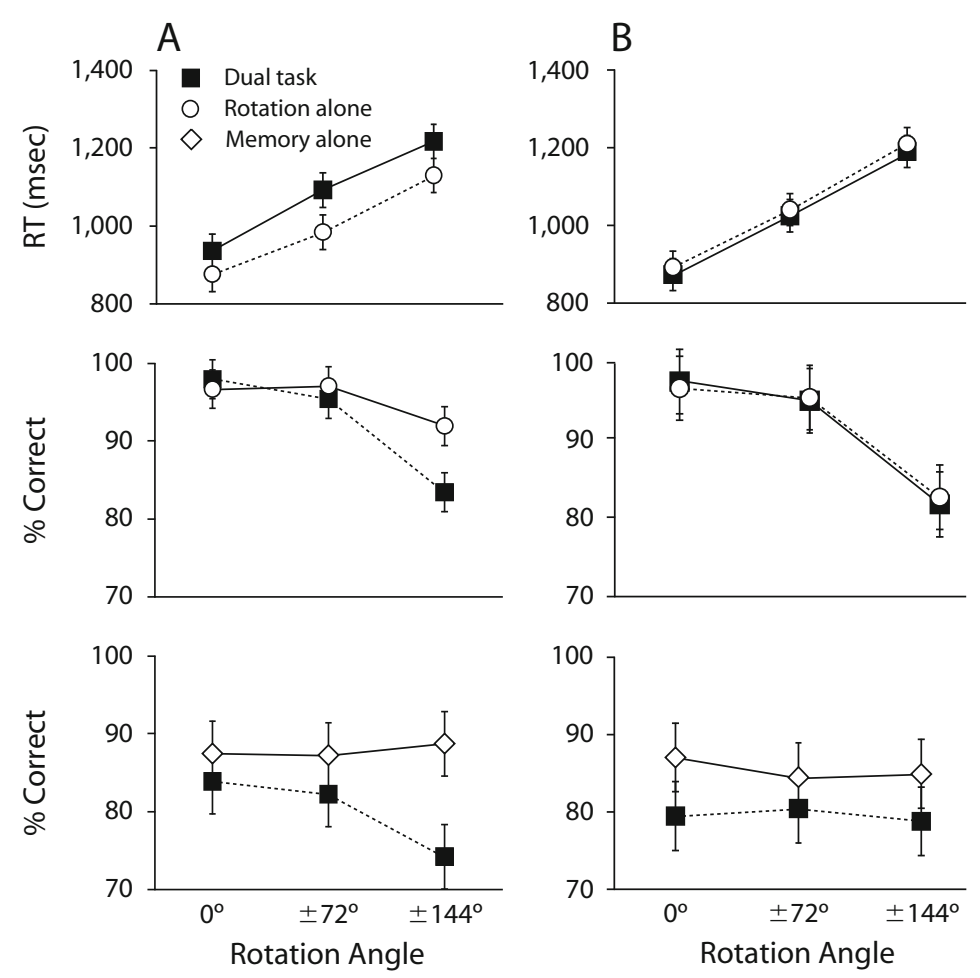

Figure 2. Results from Experiment 1 (A) and Experiment 2 (B). Top and middle rows indicate reaction time (RT) data and accuracy data from the mental rotation task when subjects were performing the rotation task with memory load (dual task) versus when they were performing the rotation task alone (single task). The bottom row indicates accuracy of the memory load task across when subjects were performing both mental rotation and working memory tasks (dual task) versus when they were performing the working memory task alone (single task). Error bars represent within-subjects $95 \%$ confidence intervals.

each other. This interference was observed in rotationdependent declines in the accuracy of both the mental rotation task and the working memory task. Interestingly, the rate of mental rotation was not slowed in the dual-task condition. We conducted a follow-up experiment in which we instructed the participants to be as accurate as possible in the mental rotation task - even if this resulted in slower responses - and exactly the same pattern of results was obtained. This finding suggests that the rate of rotation cannot easily be slowed, resulting in lower accuracy when the rotation process is challenged by a concurrent task.

Recall that interference of this nature was not observed when a visual search task was performed during the retention interval of this same working memory task (Woodman et al., 2001). That is, neither the rate nor the accuracy of visual search was impaired when search was performed during the retention interval of the memory task, and only a small and set size-independent reduction in memory performance was observed in the dual-task condition in comparison with the memory-alone condition. Thus, the pattern of interference depends on the nature of the task being performed during the retention interval. In contrast, substantial set size-dependent interference was observed when the visual search task was performed during the re- tention interval of a spatial working memory task, indicating that visual search does rely on memory for spatial location (Woodman \& Luck, 2004). In Experiment 2, we similarly asked whether mental rotation relies on spatial working memory.

\section{EXPERIMENT 2}

\section{Method}

The method of Experiment 2 was the same as that of Experiment 1 except as noted. Fifteen new participants were recruited (3 male; 10 right-handed). The stimuli and procedure are illustrated in Figure 1B. The memory stimuli consisted of four white dots $\left(0.16^{\circ} \times 0.16^{\circ}\right)$, one on each edge of the central placeholder box. Each dot was positioned randomly between $0^{\circ}$ and $0.74^{\circ}$ away from the midpoint of the edge. On change trials, one dot in the test display was displaced by $0.98^{\circ}$ from its original position. ${ }^{1}$

\section{Results and Discussion}

Figure 2B illustrates the results. The rate of mental rotation was $460^{\circ} / \mathrm{sec}$. No evidence of interference was observed in mental rotation speed or accuracy. The main effect of the degree of rotation was statistically significant for both mental rotation RT $[F(2,28)=67.9, p<$ $.001]$ and mental rotation accuracy $[F(2,28)=23.7, p<$ 
.001]. However, neither mental rotation RT nor accuracy showed a significant main effect of condition or a significant interaction between condition and degree of rotation (all $F_{\mathrm{S}}<1$ ).

The only sign of interference was a reduction in memory accuracy in the dual-task condition in comparison with the memory-alone condition, but this effect did not increase as the degree of rotation increased. This effect was small: Using the Pashler-Cowan $K$ equation (Cowan et al., 2005), we estimated that this effect was equivalent to a decline of 0.53 object's worth of information in the dual-task condition compared with the memory-alone condition. The main effect of condition was significant $[F(1,14)=6.0, p<.05]$, but neither the rotation main effect nor the condition $\times$ rotation interaction was significant (both $F \mathrm{~s}<1$ ). Because this effect was independent of the degree of rotation, it presumably reflects a general dual-task cost rather than a direct effect of the rotation process on the working memory representations. In contrast, memory performance declined in a rotation-dependent manner in Experiment 1. Thus, this experiment indicates that mental rotation and spatial working memory do not interfere much with each other.

To provide statistical support for the different patterns of interference observed in Experiments 1 and 2, we combined the data from both experiments into a single ANOVA for each dependent variable (rotation speed, rotation accuracy, and memory accuracy) with a betweensubjects factor of experiment and within-subjects factors of condition (single task vs. dual task) and degree of rotation. For mental rotation accuracy, the presence of a condition $\times$ rotation interaction for the object memory task (Experiment 1) but not for the spatial memory task (Experiment 2) yielded a significant three-way interaction between experiment, condition, and degree of rotation $[F(2,56)=4.07, p<.05]$. The same general pattern was also present for memory accuracy, but it did not approach statistical significance $(p>.1)$, which may reflect the low statistical power of a noncrossover three-way interaction involving a between-subjects factor. Nonetheless, the significant three-way interaction for mental rotation accuracy provides clear statistical support for the claim that the maintenance of color information in working memory interferes with mental rotation more than the maintenance of spatial information. Moreover, this finding cannot be a result of differences in the difficulty of the spatial and object memory tasks because overall memory accuracy was nearly identical across the two experiments.

\section{GENERAL DISCUSSION}

These two experiments provide clear evidence that the mental rotation of letters involves the object working memory storage subsystem, but not the spatial working memory storage subsystem. That is, substantial rotationdependent interference was observed when observers attempted to rotate a letter while concurrently holding object colors in memory, but minimal interference was observed when locations were being held in memory.
This is the converse of the pattern found when a visual search task was performed while object or location information was being held in working memory (Woodman \& Luck, 2004; Woodman et al., 2001). The combination of findings across experiments thus provides the equivalent of a double dissociation: Mental rotation leads to interference when combined with object working memory but not with spatial working memory, whereas visual search leads to interference when combined with spatial working memory, but not with object working memory. Double dissociations are particularly powerful sources of evidence, because they rule out explanations that are based on factors such as differential sensitivity to interference.

In the present case, the conclusion of a double dissociation is made on the basis of the combined results of several individual experiments with different subjects and slightly different methods. However, many of the individual components of this apparent double dissociation have been replicated with different subjects and with methodological variations (Hyun \& Luck, 2006; Woodman, 2002; Woodman et al., 2001), so this pattern appears to be robust.

Neuroimaging studies of mental rotation have consistently shown that dorsal stream areas exhibit rotationdependent changes in neural activity (see, e.g., Carpenter et al., 1999; Gauthier et al., 2002; Jordan et al., 2001), which would seem to imply that mental rotation would involve spatial working memory rather than object working memory. However, dorsal stream neurons do not typically show the form selectivity that would be necessary to maintain object representations while they are being rotated, and the dorsal stream activity observed in neuroimaging studies presumably reflects spatial processing that is necessary to perform the rotation rather than the storage of the representation that is being rotated. Indeed, Carpenter et al. (1999) found extensive activation of both dorsal and ventral stream areas during a mental rotation task in comparison with a control task, although only the dorsal stream activation was strongly dependent on the degree of rotation. From this pattern, they hypothesized that mental rotation involves an interaction between the dorsal and ventral streams, with storage occurring in the ventral stream and rotation operations occurring in the dorsal stream. The present results provide direct support for this hypothesis.

Although an anatomical distinction between spatial and object working memory storage is clear in posterior brain areas, prefrontal areas appear to be involved in both spatial and object working memory (Postle \& D'Esposito, 1999; Rainer, Asaad, \& Miller, 1998). However, prefrontal areas appear to be involved in the control and manipulation of information in working memory rather than being the site of storage per se (see review by Ranganath \& Blumenfeld, 2005). This view of the neural substrates of working memory is consistent with the proposal that mental rotation involves the storage of information in the object working memory system even though it involves a spatial manipulation. That is, we propose that visual form information will be stored in the object memory system when it is being manipulated, even if the manipulation involves a spatial transformation. This proposal seems necessary 
because the spatial memory system presumably cannot store information about visual form.

In conclusion, mental rotation appears to involve dorsal stream spatial operations that are applied to representations stored in the ventral stream object working memory subsystem. This finding complements the conclusion that visual search involves the storage of spatial information in the dorsal stream, which is used to guide that allocation of attention within ventral stream object recognition systems. The broad pattern of results suggests that the storage and processing functions of a given visual stream can be dissociated from each other and that the processing functions of one stream can be combined with the storage functions of the other stream as needed by the current task.

\section{AUTHOR NOTE}

This research was made possible by Grants MH63001 and MH65034 from NIH. We thank Ed Vogel and Geoff Woodman for helpful discussions and Lindsay Draeger for assistance in data collection. Correspondence concerning this article should be addressed to S. J. Luck, UCDavis Center for Mind and Brain, 267 Cousteau Place, Davis, CA 95616 (e-mail: sjluck@ucdavis.edu).

\section{REFERENCES}

BAdDeley, A. D. (1986). Working memory. Oxford: Oxford University Press, Clarendon Press.

Besner, D., Davies, J., \& Daniels, S. (1981). Reading for meaning: The effects of concurrent articulation. Quarterly Journal of Experimental Psychology, 33A, 415-437.

Carpenter, P. A., Just, M. A., Keller, T. A., \& Eddy, W. (1999). Graded functional activation in the visuospatial system with the amount of task demand. Journal of Cognitive Neuroscience, 11, 9-24.

Castel, A. D., Pratt, J., \& Craik, F. I. M. (2003). The role of spatial working memory in inhibition of return: Evidence from divided attention tasks. Perception \& Psychophysics, 65, 970-981.

Cowan, N., Elliott, E. M., Saults, J. S., Morey, C. C., Mattox, S., IsMAJATUlina, A., ET AL. (2005). On the capacity of attention: Its estimation and its role in working memory and cognitive aptitudes. Cognitive Psychology, 51, 42-100.

Gauthier, I., Hayward, W. G., Tarr, M. J., Anderson, A. W., SkudLARSKI, P., \& GORE, J. C. (2002). BOLD activity during mental rotation and viewpoint-dependent object recognition. Neuron, 34, 161-171.

GoldMAN-RAKIC, P. S. (1996). Regional and cellular fractionation of working memory. Proceedings of the National Academy of Sciences, 93, 13473-13480.

HYUN, J.-S., \& LUCK, S. J. (2006). Interaction between mental rotation and working memory. Unpublished observations.

Jonides, J., Sylvester, C.-Y. C., Lacey, S. C., Wager, T. D., NichOLs, T. E., \& AwH, E. (2003). Modules of working memory. In R. H. Kluwe, G. Lüer, \& F. Rösler (Eds.), Principles of learning and memory (pp. 113-134). Cambridge, MA: Birkhäuser.

Jordan, M. I., Heinze, H. J., Lutz, K., Kanowski, M., \& JancKe, L. (2001). Cortical activations during the mental rotation of different visual objects. NeuroImage, 13, 143-152.

LogIE, R. H. (1995). Visuo-spatial working memory. Hove, U.K.: Erlbaum.

Murray, D. J. (1968). Articulation and acoustic confusability in shortterm memory. Journal of Experimental Psychology, 78, 679-684.

Postle, B. R., \& D'Esposito, M. (1999). "What"-then-"where" in visual working memory: An event-related fMRI study. Journal of Cognitive Neuroscience, 11, 585-597.

Rainer, G., AsaAd, W. F., \& Miller, E. K. (1998). Selective representation of relevant information by neurons in the primate prefrontal cortex. Nature, 393, 577-579.

Ranganath, C., \& Blumenfeld, R. S. (2005). Doubts about double dissociations between short- and long-term memory. Trends in Cognitive Sciences, 9, 374-380.

Woodman, G. F. (2002). The involvement of visual working memory in visual search. Unpublished dissertation, University of Iowa.

Woodman, G. F., \& Luck, S. J. (2004). Visual search is slowed when visuospatial working memory is occupied. Psychonomic Bulletin \& Review, 11, 269-274.

Woodman, G. F., Vogel, E. K., \& Luck, S. J. (2001). Visual search remains efficient when visual working memory is full. Psychological Science, 12, 219-224.

\section{NOTE}

1. On a substantial fraction of change trials, the position of the changed item was outside of the range of positions that were used in the sample stimuli. Consequently, participants could potentially respond fairly accurately by simply guessing "change" when the test item was in an extreme position. To demonstrate that participants do not spontaneously use this strategy, we tested 7 participants with the memory task by itself. None of the participants reported that they used this strategy, and mean performance was only $5 \%$ better when this strategy was possible (i.e., when an extreme value was present) than when it was impossible. Thus, it is very unlikely that memory performance in Experiment 2 was significantly distorted by this guessing strategy.

(Manuscript received May 30, 2005; revision accepted for publication June 7,2006 .) 